\title{
BMJ Open Relationship between exclusive breast feeding and cardiorespiratory fitness in children and adolescents: a protocol for a systematic review and meta-analysis
}

\author{
Carlos Berlanga-Macías, ${ }^{1}$ Diana P. Pozuelo-Carrascosa, ${ }^{1}$ Celia Álvarez-Bueno, ${ }^{1}$ \\ Jose Alberto Martínez-Hortelano, ${ }^{1}$ Miriam Garrido-Miguel, ${ }^{1}$ \\ Vicente Martínez-Vizcaíno ${ }^{1,2}$
}

To cite: Berlanga-Macías C, Pozuelo-Carrascosa DP., Álvarez-Bueno C, et al. Relationship between exclusive breast feeding and cardiorespiratory fitness in children and adolescents: a protocol for a systematic review and meta-analysis. BMJ Open 2018;8:e023223. doi:10.1136/ bmjopen-2018-023223

- Prepublication history for this paper is available online. To view these files, please visit the journal online (http://dx.doi. org/10.1136/bmjopen-2018023223).

Received 27 March 2018

Revised 19 June 2018

Accepted 24 September 2018

Check for updates

(c) Author(s) (or their employer(s)) 2018. Re-use permitted under CC BY-NC. No commercial re-use. See rights and permissions. Published by BMJ.

${ }^{1}$ Health and Social Research Center, Universidad de CastillaLa Mancha, Cuenca, Spain

${ }^{2}$ Facultad de Ciencias de la

Salud, Universidad Autónoma de Chile, Talca, Chile

Correspondence to Miss Diana P. Pozuelo-

Carrascosa;

dianap.pozuelo@uclm.es

\section{ABSTRACT}

Introduction Breast feeding has been considered important due to its short-term and long-term benefits on infant and maternal health. Regarding the long-term benefits, the influence of exclusive breastfeeding on cardiorespiratory fitness (CRF) during childhood and adolescence has been studied, although with controversial conclusions. This study protocol aims to provide a clear and standardised procedure for systematically reviewing the relationship between breast feeding, in terms of duration and exclusivity, and CRF in children and adolescents.

Methods and analysis This systematic review and meta-analysis protocol is reported in accordance with the Preferred Reporting Items for Systematic Reviews and Meta-Analyses Protocols. A literature search will be conducted in MEDLINE, EMBASE, Web of Science and Cochrane Library. Observational studies regarding the association between breast feeding and CRF in children and adolescents written in English or Spanish will be included. A Critical Appraisal Checklist for Analytical Cross Sectional Studies and The Newcastle-Ottawa Quality Assessment Scale for longitudinal studies will be used for quality assessment of included studies. Standardised mean differences of CRF by exclusive breastfeeding categories will be calculated as the primary outcome. Subgroup analyses and meta-regression will be performed based on the sources of heterogeneity.

Ethics and dissemination This evidence-based systematic review will summarise the relevant information on the association of exclusive breast feeding and CRF in children and adolescents. The results will be disseminated by publication in a peer-reviewed journal. Given that the data used for this systematic review will be exclusively extracted from published studies, ethical approval will not be required.

PROSPERO registration number CRD42018082642.

\section{INTRODUCTION}

The WHO recommends exclusive breast feeding during the first 6 months of life, then combining with complementary solid foods up to 2 years of age or more. ${ }^{1}$
Strengths and limitations of this study

- A systematic review and meta-analysis will be conducted derived from observational studies which limits our ability to establish casual relationships.

- Another limitation of this study is the differences in study design, sample characteristics, different methods used for measuring breast feeding or cardiorespiratory fitness (CRF), and poor quality statistical analyses which may limit comparability among included studies and decrease the quality of evidence of this systematic review and meta-analysis.

- One more limitation might be the restriction of the analysis to studies published in English and Spanish.

- An exhaustive literature search will be thoroughly carried out to identify studies aimed to assess the association between exclusive breast feeding and CRF in childhood and adolescence.

- The analysis of different sources of heterogeneity and the assessment of risk of bias of the included studies is a key point for extracting and synthesising evidence-based conclusions.

Nevertheless, in spite of these evidence-based guidelines, very low rates of compliance have been reported. Approximately, between 2007 and 2014 , just $36 \%$ of babies (0-6 months) around the world were exclusively breast fed, and in most high-income countries the prevalence is less than $20 \%$ which shows the necessity of increasing the compliance rate with the WHO recommendations. ${ }^{1-3}$ Thus, more efforts are required to disseminate the importance of implementing this type of feeding during the first 2 years of life. ${ }^{2}$

Several studies have shown the short-term and long-term benefits of breast feeding on infant and maternal health. When breast feeding is primarily exclusive, it protects against common diseases during childhood, such as respiratory infections, otitis media or diarrhoea. Additionally, data 
suggest that exclusive breast feeding might decrease the probability of infectious mortality and dental malocclusions, and reduces the risk of sudden infant death syndrome. ${ }^{3}{ }^{4}$ Neuropsychological benefits for infants, better motor development in childhood and more intelligence during young adult life are a few examples of the long-term advantages related to breast feeding, notwithstanding that further investigations are necessary. ${ }^{5-7}$ Moreover, breast feeding acts as a protective factor against severe cardiovascular risk factors in children: overweight, obesity, hypertension, high fasting plasma glucose, high blood concentration of triglycerides or low blood concentrations of high-density lipoprotein cholesterol which all have deleterious effects in adulthood. ${ }^{8-11}$ Regarding maternal health, breast feeding has been shown to accelerate postpartum weight loss, and it is involved in the risk reduction of breast and ovarian cancer, and type 2 diabetes, among others. ${ }^{912}$

Cardiorespiratory fitness (CRF) is a powerful and effective indicator of cardiovascular health in the early stages of life ${ }^{13}$ besides being related to a lower risk of all-cause mortality in adulthood. ${ }^{14-16}$ In fact, CRF improves blood pressure and decreases abdominal adiposity, and is considered a protective factor for metabolic syndrome, cardiovascular illnesses and cancer occurrence in adult life. ${ }^{141718}$ It is important to highlight that the last two are among the 10 leading causes of death in the world. ${ }^{19}$ Likewise, the death risk is twice higher in unfit people, those who do not meet the 2008 Physical Activity Guidelines, independently of body mass index (BMI), as was shown in a recent meta-analysis. ${ }^{2021}$

CRF is influenced by several genetic, biological, social, environmental and lifestyle factors, ${ }^{22}$ including breast feeding. Recently, many studies have analysed the importance of breast feeding in CRF and other fitness components in children and adolescents. ${ }^{23-27}$ However, their conclusions are controversial, in such a way that while some of them did not report a relationship between breast feeding and CRF, ${ }^{23} 2527$ others found that duration of exclusive breast feeding is positively related to later CRF. ${ }^{24}{ }^{26}$ Human milk components, such as polyunsaturated fatty acids, prostaglandin $\mathrm{J} 2$ and adipokines might explain the positive influence of breast feeding on CRF. ${ }^{28}$ Therefore, further research is necessary to obtain stronger conclusions on the possible effects of breast feeding on CRF.

Thus, the aim of this novel methodological study protocol is to provide a clear procedure for systematically reviewing the relationship between breast feeding, in terms of duration and exclusivity, and CRF in children and adolescents.

\section{OBJECTIVE}

The purpose of this study protocol is to report a standardised and clear methodology to carry out a systematic review and meta-analysis aimed to assess the relationship between breast feeding, in terms of duration and exclusivity, and CRF in children and adolescents aged 4-18 years.

\section{METHODS AND ANALYSIS}

This systematic review and meta-analysis protocol has been registered in PROSPERO. In addition, this protocol adheres to the Preferred Reporting Items for Systematic Reviews and Meta-Analyses Protocols (PRISMA-P) ${ }^{29}$ The systematic review and meta-analysis will be guided by the Meta-analysis Of Observational Studies in Epidemiology (MOOSE) statement and Cochrane Collaboration Handbook recommendations. ${ }^{30} 31$

\section{Inclusion/exclusion criteria for study selection}

Studies concerning the association between breast feeding, in terms of duration and exclusivity, and CRF in children and adolescents which meet all inclusion criteria will be included in the systematic review: (1) participants: children aged 4-12 years and adolescents aged 12-18 years; (2) exposure: exclusive breast feeding and duration (even if it is little or no exclusive breast feeding); (3) outcome: CRF determined by standardised tests whose scientific rationale, validity and reliability has been previously published, such as $20 \mathrm{~m}$ shuttle run test, cardiorespiratory exercise test (by treadmill or ergometer bike) or heart rate; and (4) study design: observational studies (cross-sectional or follow-up studies).

Studies will be excluded when: (1) they are not written in English or Spanish; and (2) they include participants older than 18 years. Studies will also be excluded when the target population was specifically: (1) children with severe physical limitations that could impede CRF measurement; and (2) they refer to a particular population group, such as professional and high-performance athletes.

\section{Search strategy}

A literature search will be conducted, and studies will be identified through a combination of resources. First, a systematic search will be performed in MEDLINE (via PubMed), EMBASE, Web of Science and Cochrane Library from their inception. The search strategy will combine boolean operators from the following relevant concepts: breastfeeding, feeding, breast, 'breast suckling', suckling, 'exclusive breastfeeding', 'cardiorespiratory fitness', fitness, 'VO2 max', 'physical fitness', 'aerobic capacity', 'cardio fitness', children, adolescents, childhood, scholars and school-aged (table 1). Previous systematic reviews and meta-analyses, and relevant references of the selected studies will be reviewed to identify additional sources. Study records will be organised using the Mendeley Reference Manager.

\section{Selection of studies and data extraction}

All titles and abstracts of the retrieved articles will be independently screened by two researchers to identify eligible studies for this systematic review, according 
Table 1 Search strategy for the MEDILINE database

\begin{tabular}{lll} 
Breastfeeding & 'cardiorespiratory & children \\
OR & fitness' & OR \\
feeding & OR & adolescents \\
OR & fitness & OR \\
breast & OR & childhood \\
OR & 'VO2 max' & OR \\
'breast & AND OR & AND \\
suckling' & 'physical fitness' & OR \\
OR & OR & School- \\
suckling & 'aerobic capacity' & aged \\
OR & OR & \\
'exclusive & 'cardio fitness' \\
breastfeeding' & & \\
\hline
\end{tabular}

to the inclusion criteria. The studies that do not meet the eligibility criteria will be excluded. Abstracts not providing enough information regarding the inclusion/ exclusion criteria will be selected for full-text evaluation. Two reviewers will examine the included and excluded studies to verify the reason for each decision. Inconsistencies in data collection will be solved by consensus. A third researcher will be asked when consensus is not reached. The process of identifying, screening and including/ excluding articles will be shown using the PRISMA ${ }^{29}$ flow chart (figure 1).

Additionally, the full text of the identified studies will be examined to extract the following data: (1) first author's name, (2) publication year, (3) country, (4) study design, (5) number of children in each sample, (6) age of children at evaluation, (7) breast feeding (categories and number of participants in each group) and (8) CRF (tools and measurements used for the assessment of participants). The authors of the included studies will be contacted when a lack of information is detected.

\section{Assessment of risk of bias}

Before assessing the methodological quality of the included studies, two reviewers will be blinded to the authors, title and year of publication of the studies. Then, standardised checklists for observational studies will be used. To assess the quality of cross-sectional studies, a Critical Appraisal Checklist for Analytical Cross Sectional Studies from The Joanna Briggs Institute will be applied. ${ }^{32}$ It consists of eight items scoring as 'yes', 'no', 'unclear' and 'not applicable'. The results of this appraisal will be used to show the possibility of bias in the design, conduct and analysis of each study.
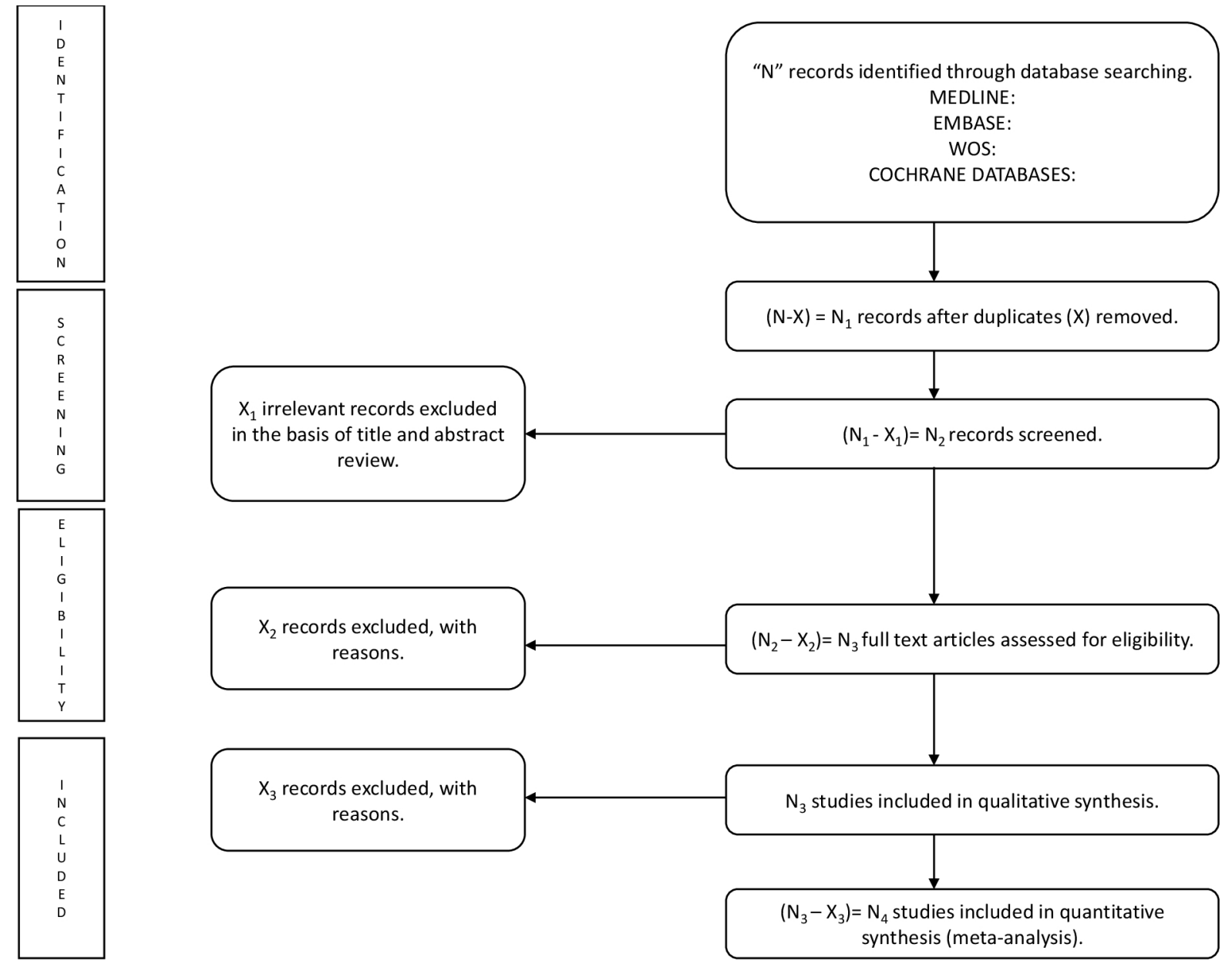

Figure 1 Preferred Reporting Items for Systematic Reviews and Meta-Analyses flow diagram of identification, screening, eligibility and inclusion of studies. 
Table 2 Characteristics of studies included in the systematic review and/or meta-analysis

\begin{tabular}{|c|c|c|c|c|c|c|c|c|}
\hline \multirow[b]{2}{*}{ Reference } & \multirow[b]{2}{*}{ Country } & \multirow{2}{*}{$\begin{array}{l}\text { Study } \\
\text { design }\end{array}$} & \multicolumn{2}{|l|}{ Population } & \multicolumn{2}{|l|}{ Breast feeding } & \multicolumn{2}{|l|}{ CRF } \\
\hline & & & Sample size & Sample age & Categories & $\mathbf{n}$ & Tool & Measurement \\
\hline $\begin{array}{l}\text { First author's } \\
\text { name and year } \\
\text { of publication }\end{array}$ & Country & $\begin{array}{l}\text { Design } \\
\text { of the } \\
\text { study }\end{array}$ & $\begin{array}{l}\text { Number of } \\
\text { participants }\end{array}$ & $\begin{array}{l}\text { Age of } \\
\text { participants } \\
\text { (years) }\end{array}$ & $\begin{array}{l}\text { Duration periods } \\
\text { of exclusive } \\
\text { breast feeding }\end{array}$ & $\begin{array}{l}\text { Number } \\
\text { participants } \\
\text { in each } \\
\text { breastfeeding } \\
\text { category }\end{array}$ & $\begin{array}{l}\text { Instrument } \\
\text { used to } \\
\text { measure } \\
\text { CRF }\end{array}$ & $\begin{array}{l}\text { Measure used to } \\
\text { express CRF }\end{array}$ \\
\hline
\end{tabular}

CRF, cardiorespiratory fitness.

The Newcastle-Ottawa Quality Assessment Scale ${ }^{33}$ will be used to assess the quality of longitudinal studies in meta-analyses, including case-control and cohort studies. This scale includes eight items grouped in three categories: (1) selection, (2) comparability and (3) exposure or outcome (depending on whether it is case-control or cohort studies, respectively). In the evaluation process, each study can obtain one star for each item within the selection and exposure categories, and a maximum of two stars in the comparability category.

Any conflicts over the assessment of quality will be solved by consensus. A third researcher will be consulted if a consensus cannot be reached.

\section{Statistical analysis}

Authors will summarise the main characteristics of included studies and any relevant question according to the aim of this systematic review through table 2, in which the study's characteristics, methods, description of population and relevant issues related to outcomes will be included. After data extraction, it will be determined if a meta-analysis is possible. If possible, a meta-analysis will be carried out using STATA V.14 software to compute pooled effect size (ES) estimates with $95 \%$ CIs.

Adjusted or unadjusted models, depending on the data extracted, will be used to compare the level of CRF when children and adolescents have never been breast fed, as reference, with CRF when children and adolescents have been exclusively breast fed for any time. A fixed-effects model ${ }^{34}$ will be used if there is no evidence of heterogeneity; otherwise, a random-effects model ${ }^{35}$ will be used. The standardised mean difference will be calculated for each breastfeeding category as an estimate of $\mathrm{ES}^{36}{ }^{36} \mathrm{using}$ Cohen's index as the ES statistic, if possible, in which a positive ES value indicates an increase of CRF in favour of breastfeeding versus the never breastfed group.

The heterogeneity of results across studies will be evaluated using the $\mathrm{I}^{2}$ statistic that is considered as: not important $(0 \%-40 \%)$, moderate $(30 \%-60 \%)$, substantial $(50 \%-90 \%)$ and considerable $(75 \%-100 \%)$; the corresponding $\mathrm{p}$ values will also be considered. ${ }^{37}$ Additionally, publication bias will be assessed using a funnel plot, according to the method proposed by Egger et al. ${ }^{38}$

Finally, if a meta-analysis is not possible, due to a lack of quantitative information or if there is considerable heterogeneity between studies, a systematic review with descriptive analysis will be conducted.

\section{Subgroup analyses and meta-regression}

Subgroup analyses and meta-regression will be conducted on the main factors that could cause heterogeneity: (1) type of study design (longitudinal or cross-sectional); (2) country; (3) characteristics of population, such as age (children aged 4-12 years and adolescents aged 12-18 years); (4) breastfeeding classification (never, less than 6 months and more than 6 months) ${ }^{1}$; (5) aspects related to CRF, such as type of tool used (20 m shuttle run test (20 $\mathrm{m}$ SRT), cycle-ergometer, treadmill test) and measurement reported (VO2 max, stage from $20 \mathrm{~m} \mathrm{SRT,} \mathrm{watts);}$ and (6) covariates included in the original studies (birth weight, BMI, physical activity, among others). Additionally, the methodological quality of included studies, as reported previously, will be taken into account for additional subgroup analyses.

\section{Sensitive analyses}

Sensitivity analyses will be carry out, removing the included studies one by one from the pooled analyses, to assess the robustness of summary estimates and to detect if any singular study accounts for a large proportion of heterogeneity.

\section{Patient and public involvement}

Patients and/or public will not be involved in the conducting of this study. However, once our results are disseminated by scientific publications, it will be shared through social networks and infant feeding-related population groups, in such a way that our conclusions would influence breastfeeding-related mother's behaviour.

\section{DISCUSSION}

Complying with WHO recommendations on breast feeding has important benefits for infant and maternal health. With long-term benefits, several studies have evaluated the influence of exclusive breast feeding over CRF in childhood and adolescence. However, there is no consensus on the real effects, since results and conclusions were controversial. ${ }^{23-27}$ Therefore, we will conduct a systematic review and meta-analysis, if possible, to show a general view of the current literature, as well as further improve research regarding exclusive breast feeding and CRF. For that purpose, the protocol of this systematic review provides a clear structure for extraction and synthesis of relevant information. 
Another aspect to consider with the implementation of the systematic review is to know if strictly complying with WHO recommendations increases CRF during childhood and adolescence which is a powerful health marker at these ages. Likewise, we will report if breast feeding could mitigate the deleterious effect, over CRF, of several factors regarding intrauterine growth, such as low birth weight. ${ }^{39}$ If this association is demonstrated through synthesis of evidence, breast feeding could acquire a greater interest in the public health of children and adolescents.

Additionally, since different types of observational studies will be considered for inclusion (cross-sectional and follow-up studies), we will use two tools for quality assessment: (1) a Critical Appraisal Checklist for Analytical Cross Sectional Studies ${ }^{32}$ and (2) The Newcastle-Ottawa Quality Assessment Scale for longitudinal studies. ${ }^{33}$

Furthermore, any sources of heterogeneity of studies included, such as study design, country, sample characteristics (size and age range), confounding factors and tool used for measurement of breast feeding and CRF, will be considered in this review using random-effects meta-regression. ${ }^{40}$ Subgroup analyses and sensitivity analyses will be conducted to control and determine if some included studies account for a proportion of heterogeneity.

Potential limitations are inherent in conducting systematic reviews and meta-analyses: publication bias, information bias, poor statistical analyses, poor methodological quality and inadequate reporting of methods and findings of the included studies. Another limitation might be the restriction of the analysis to studies published in English and Spanish, since it could introduce a language bias and overestimate the magnitude of the possible associations. Therefore, it is important to properly synthesise the information available in the manuscripts included. This task will be conducted independently by two reviewers, and disagreements will be resolved by consensus. Likewise, the MOOSE statement and Cochrane Collaboration Handbook recommendations will be followed. ${ }^{30} 31$

In summary, due to a lack of agreement and robust conclusions about the relationship between exclusive breast feeding and CRF in children and adolescents, it seems logical and important to carry out a systematic review and meta-analysis.

\section{ETHICS AND DISSEMINATION}

Because the data used for this systematic review will be exclusively extracted from published studies, ethical approval and informed consent of patients will not be required. The resulting findings of this study could help us develop high-quality recommendations about the longterm role of breast feeding, such as: (1) breast feeding as an enabling factor for the enhancement of CRF in children and adolescents and (2) breast feeding as a resource for mitigating the negative effect of different covariates on CRF. Additionally, it could provide updated epidemiological data about the prevalence of exclusive breast feeding. This study could also have important public health implications, as health professionals are key actors to promote and transfer knowledge about breast feeding in the general population. Finally, this protocol is considered as a necessary step for solidifying the methodological structure to conduct an evidencebased systematic review and meta-analysis regarding the association between exclusive breast feeding and CRF in young populations.

Contributors VM-V and CB-M designed the study. VM-V was the principal investigator and guarantor. VM-V and CB-M were the main coordinators of the study. DPP-C, CA-B, JAM-H, MG-M and VM-V conducted the study. CB-M, DPP-C and $C A-B$ gave statistical and epidemiological support. CB-M wrote the article with the support of DPP-C and VM-V. All authors reviewed and approved the final version of the manuscript.

Funding CB-M, DPP-C and MG-M are supported by grants from the Spanish Ministry of Education, Culture and Sport (FPU16/02380, FPU14/01370 and FPU15/03847, respectively). JAM-H is supported by a grant from the University of Castilla-La Mancha (6A2400/NL65671).

Competing interests None declared.

Patient consent Not required.

Provenance and peer review Not commissioned; externally peer reviewed.

Open access This is an open access article distributed in accordance with the Creative Commons Attribution Non Commercial (CC BY-NC 4.0) license, which permits others to distribute, remix, adapt, build upon this work non-commercially, and license their derivative works on different terms, provided the original work is properly cited, appropriate credit is given, any changes made indicated, and the use is non-commercial. See: http://creativecommons.org/licenses/by-nc/4.0/.

\section{REFERENCES}

1. World Health Organization. Infant and young child feeding. Geneva: WHO, 2017.

2. Rollins NC, Bhandari N, Hajeebhoy N, et al. Why invest, and what it will take to improve breastfeeding practices? Lancet 2016;387:491-504.

3. Victora CG, Bahl R, Barros AJ, et al. Breastfeeding in the 21st century: epidemiology, mechanisms, and lifelong effect. Lancet 2016;387:475-90.

4. Hauck FR, Thompson JM, Tanabe KO, et al. Breastfeeding and reduced risk of sudden infant death syndrome: a meta-analysis. Pediatrics 2011;128:103-10.

5. Tozzi AE, Bisiacchi P, Tarantino V, et al. Effect of duration of breastfeeding on neuropsychological development at 10 to 12 years of age in a cohort of healthy children. Dev Med Child Neurol 2012;54:843-8.

6. Michels KA, Ghassabian A, Mumford SL, et al. Breastfeeding and motor development in term and preterm infants in a longitudinal US cohort. Am J Clin Nutr 2017;106:1456-62.

7. Mortensen EL, Michaelsen KF, Sanders SA, et al. The association between duration of breastfeeding and adult intelligence. JAMA 2002;287:2365-71.

8. Wisnieski L, Kerver J, Holzman C, et al. Breastfeeding and Risk of Metabolic Syndrome in Children and Adolescents: A Systematic Review. J Hum Lact 2018;34.

9. Mastroeni MF, Mastroeni S, Czarnobay SA, et al. Breast-feeding duration for the prevention of excess body weight of motherchild pairs concurrently: a 2-year cohort study. Public Health Nutr 2017;20:2537-48.

10. Breij LM, Abrahamse-Berkeveld M, Acton D, et al. Impact of Early Infant Growth, Duration of Breastfeeding and Maternal Factors on Total Body Fat Mass and Visceral Fat at 3 and 6 Months of Age. Ann Nutr Metab 2017;71(3-4):203-10.

11. Arenz S, Rückerl R, Koletzko B, et al. Breast-feeding and childhood obesity - a systematic review. Int J Obes 2004;28:1247-56.

12. Anstey $\mathrm{EH}$, Shoemaker ML, Barrera CM, et al. Breastfeeding and Breast Cancer Risk Reduction: Implications for Black Mothers. Am J Prev Med 2017;53:S40-S46.

13. Ruiz JR, Cavero-Redondo I, Ortega FB, et al. Cardiorespiratory fitness cut points to avoid cardiovascular disease risk in children and adolescents; what level of fitness should raise a red flag? 
A systematic review and meta-analysis. Br J Sports Med 2016;50:1451-8

14. Kodama S, Saito K, Tanaka S, et al. Cardiorespiratory fitness as a quantitative predictor of all-cause mortality and cardiovascular events in healthy men and women: a meta-analysis. JAMA 2009;301:2024-35.

15. Blair SN, Kampert JB, Kohl HW, et al. Influences of cardiorespiratory fitness and other precursors on cardiovascular disease and all-cause mortality in men and women. JAMA 1996;276:205-10.

16. Blair SN, et al. Physical Fitness and All-Cause Mortality. JAMA 1989;262:2395-401.

17. Ruiz JR, Castro-Piñero J, Artero EG, et al. Predictive validity of health-related fitness in youth: a systematic review. Br J Sports Med 2009;43:909-23.

18. Vainshelboim B, Müller J, Lima RM, et al. Cardiorespiratory fitness and cancer incidence in men. Ann Epidemiol 2017;27:442-7.

19. World Health Organization. The top 10 causes of death. Geneva: WHO, 2017.

20. Barry VW, Baruth M, Beets MW, et al. Fitness vs. fatness on all-cause mortality: a meta-analysis. Prog Cardiovasc Dis 2014;56:382-90.

21. US Department of Health and Human Services. Physical Activity Guidelines for Americans. Washington DC: US Department of Health and Human Services, 2008.

22. Ortega FB, Ruiz JR, Castillo MJ, et al. Physical fitness in childhood and adolescence: a powerful marker of health. Int $J$ Obes 2008;32:1-11.

23. Artero EG, Ortega FB, España-Romero V, et al. Longer breastfeeding is associated with increased lower body explosive strength during adolescence. J Nutr 2010;140:1989-95.

24. Labayen I, Ruiz JR, Ortega FB, et al. Exclusive breastfeeding duration and cardiorespiratory fitness in children and adolescents. Am J Clin Nutr 2012;95:498-505.

25. Lawlor DA, Cooper AR, Bain C, et al. Associations of birth size and duration of breast feeding with cardiorespiratory fitness in childhood: findings from the Avon Longitudinal Study of Parents and Children (ALSPAC). Eur J Epidemiol 2008;23:411-22.

26. Vafa M, Heshmati J, Sadeghi H, et al. Is exclusive breastfeeding and its duration related to cardio respiratory fitness in childhood? J Matern Fetal Neonatal Med 2016;29:461-5.

27. Zaqout M, Michels N, Ahrens W, et al. Associations between exclusive breastfeeding and physical fitness during childhood. Eur J Nutr 2018;57:545-55.
28. Verier C, Meirhaeghe A, Bokor S, et al. Breast-feeding modulates the influence of the peroxisome proliferator-activated receptorgamma (PPARG2) Pro12Ala polymorphism on adiposity in adolescents: The Healthy Lifestyle in Europe by Nutrition in Adolescence (HELENA) cross-sectional study. Diabetes Care 2010;33:190-6.

29. Moher D, Shamseer L, Clarke M, et al. Preferred reporting items for systematic review and meta-analysis protocols (PRISMA-P) 2015 statement. Syst Rev 2015;4:1.

30. Stroup DF, Berlin JA, Morton SC, et al. Meta-analysis of observational studies in epidemiology: a proposal for reporting. Meta-analysis Of Observational Studies in Epidemiology (MOOSE) group. JAMA 2000;283:2008-12.

31. The Cochrane Collaboration. In: Higgins JP, Green S, Cochrane handbook for systematic reviews of interventions. 5.1.0, 2011. http:/ handbook.cochrane.org. (updated Mar 2011).

32. Moola S, Munn Z, Tufanaru C, et al. Chapter 7: Systematic reviews of etiology and risk. In: Aromataris E, Munn Z, eds. Joanna Briggs Institute Reviewer's Manual. The Joanna Briggs Institute, 2017. Available from. https://reviewersmanual.joannabriggs.org/.

33. Wells GA, Shea B, O'Connell D, et al. The Newcastle-Ottawa Scale (NOS) for Assessing the Quality of Nonrandomised Studies in Metaanalyses. Ottawa: Ottawa Hospital Research Institute. http:// www.ohri.ca/programs/clinical_epidemiology/oxford.asp. (accessed 31 May 2018).

34. Leonard T, Duffy JC. A Bayesian fixed effects analysis of the Mantel-Haenszel model applied to meta-analysis. Stat Med 2002;21:2295-312.

35. DerSimonian R, Kacker R. Random-effects model for meta-analysis of clinical trials: an update. Contemp Clin Trials 2007;28:105-14.

36. Lipsey M, Wilson D. Practical meta-analysis. Thousand Oaks, CA Sage Publications, 2001

37. Higgins JP, Thompson SG. Quantifying heterogeneity in a metaanalysis. Stat Med 2002;21:1539-58.

38. Egger M, Davey Smith G, Schneider M, et al. Bias in meta-analysis detected by a simple, graphical test. BMJ 1997;315:629-34.

39. van Deutekom AW, Chinapaw MJM, Vrijkotte TGM, et al. The association of birth weight and infant growth with physical fitness at 8-9 years of age - the ABCD study. Int J Obes 2015;39:593-600.

40. Thompson SG, Sharp SJ. Explaining heterogeneity in meta-analysis: a comparison of methods. Stat Med 1999;18:2693-708. 